\title{
Induced Spirals in Polyethylene Terephthalate Films Irradiated with Ar Ions with an Energy of $70 \mathrm{MeV}$
}

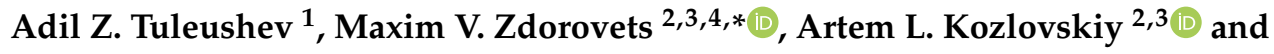 \\ Fiona Erica Harrison ${ }^{2}$ \\ 1 Flerov Laboratory of Nuclear Reactions, Joint Institute for Nuclear Research, Dubna 141980, Moscow Region, \\ Russia; adilzht@mail.ru \\ 2 Engineering Profile Laboratory, L.N. Gumilyov Eurasian National University, Nur-Sultan 010008, \\ Kazakhstan; kozlovskiy.a@inp.kz (A.L.K.); fiona_e_harrison@hotmail.com (F.E.H.) \\ 3 The Institute of Nuclear Physics, Almaty 050032, Kazakhstan \\ 4 Department of Intelligent Information Technologies, Ural Federal University, Yekaterinburg 620075, Russia \\ * Correspondence: mzdorovets@inp.kz
}

Received: 25 April 2020; Accepted: 25 May 2020; Published: 27 May 2020

check for updates

\begin{abstract}
This paper presents the results of a study of the ordering in polyethylene terephthalate (PET) film induced by $\mathrm{Ar}^{8+}$ ions with an irradiation fluence of $2 \times 10^{12}$ ions $/ \mathrm{cm}^{2}$, and of the temporal stability of the induced ordering in the irradiated sample, over a three month period. Immediately after irradiation, sharp new reflections not seen at lower fluences were observed in X-ray diffraction patterns, with angular positions of $2 \theta=9-10^{\circ}$ and $19^{\circ}$ and variable azimuthal intensities. X-ray reflections, previously observed at lower fluences, were also seen: at $2 \theta=26^{\circ}$ and $23^{\circ}$, associated with PET crystallites, and at $2 \theta=5-12^{\circ}$, associated with induced ordering in the amorphous zone. Aging of the irradiated sample led to significant growth of the ordering region in the amorphous zone for angles up to $2 \theta<15^{\circ}$, as well as to dissipation and blurring of the new diffraction reflections at $2 \theta=9-10^{\circ}$ and $2 \theta=19^{\circ}$ and the formation of a new diffraction ring reflection in the range $2 \theta=11-16^{\circ}$. The azimuthal distribution of diffraction reflection intensities immediately after irradiation displays a clear oblique cross located predominantly along lines at angles of $\pi / 4$ with respect to the direction of the texture of the PET film, indicating the formation of spiral structures based on the molecular strands of PET. Our experimental results lead us to conclude that the formation of coherent scattering areas in the amorphous region at $2 \theta<15^{\circ}$ is due to intra-chain rotations of benzene-carboxyl subunits of repeat units of the PET chain molecules interacting with the residual electric field of a single latent track; whereas the formation of spiral structures is due to the inter-chain interaction of these preordered asymmetric subunits under the influence of the electric fields from overlapping latent tracks.
\end{abstract}

Keywords: polyethylene terephthalate; latent tracks of heavy ions; irradiation-induced spirals; intra-chain and inter-chain ordering

\section{Introduction}

Over the last three decades, the heavy ion irradiation of solid materials including polymers has been the subject of extensive research, studying both the fundamental interaction mechanisms and the opportunities for real-world applications. This is evidenced by a large number of articles summarized both in reviews [1,2] and major monographs [3]. Irradiation of polymers with swift ions leads to various irreversible effects, such as amorphization and destruction [4,5], surface modification [6-8], and chemical cross-linking of polymers [9-11]. The irreversibility of these effects is due to them being caused by highly energetic $\delta$-electrons knocked out from the track core by the irradiating ions and cascades of secondary electrons formed in turn by these $\delta$-electrons [9-13]. The stochastic nature of 
these processes means that these changes in the molecular structure of irradiated polymers inside the latent tracks are also stochastic [12-14], so cannot cause molecular ordering (which, as far as we are aware, has never been observed), either directly or through the aging processes in irradiated plastic films.

Ordered changes have, however, been observed in the radial electron density of the latent track. In the experiments of Abu Saleh et al. [15], it was found that the radial electron density increased with increasing radius, with the density in the inner core of the latent track (at radii less than $3 \mathrm{~nm}$ ) being only $20 \%$ of that in the shell of the latent track. It follows from Maxwell's equations that there is a radially inhomogeneous electric field across the latent track.

The work of Wang et al. [16], showed that there is a residual radial electric field in latent tracks after irradiation. The stability of this electric field over time is determined by the properties of the irradiated material and can be very long in the case of electrets [17]. The electric field can be significantly affected by UV illumination, as shown by Wang et al. [16], who invented a special technique for processing polyethylene terephthalate (PET) film known as the 'track-UV technique'. This technique is very close to the technology used to prepare ion irradiated films for etching [18] and is based on the general sensitivity of PET to UV illumination [19].

The presence of a residual radial electric field in the latent track in PET is the cause of the induced ordering of benzene-carboxyl subunits of repeat units of polymer molecules seen in [20]. It is well-known that these subunits have the ability to rotate relative to the backbone of the molecule: the rotational properties of molecular subunits forms the basis of the field of stereochemistry, see e.g., [21].

In this paper, we report a novel ordering effect in PET film irradiated with swift $\mathrm{Ar}^{8+}$ ions at a higher fluence (of $2 \times 10^{12} \mathrm{ions} / \mathrm{cm}^{2}$ ) than used in [20]. This new structural effect is expressed in sharp X-ray diffraction peaks, at $2 \theta=9-10^{\circ}$ and $2 \theta=19^{\circ}$, with variable azimuthal intensity. As seen previously, the increase in fluence causes a reduction in the average intensity of the crystalline phase diffraction reflections at $2 \theta=26^{\circ}$ and $2 \theta=23^{\circ}$. At this higher fluence, however, both of these previously isotropic azimuthal distributions now exhibit non-isotropic behavior. In the amorphous phase, we again see an area of induced ordering along the direction of the molecular texture of the polymer with an intensity that coincides with that seen at the lower fluence of $1 \times 10^{12} \mathrm{ions} / \mathrm{cm}^{2}$. These new effects go beyond those seen at lower fluences, but since these higher fluences are achieved by longer periods of exposure to the same incident beam, these effects build cumulatively on those of lower fluences.

The appearance of these new features in the X-ray diffractograms suggests that there has been a change in the nature of structural transformations in the polymer under the influence of this higher irradiation dose. In order to explore the nature of these structural transformations, and to confirm that the changes we observed are not an experimental artifact, we conducted a study of their evolution over time. After 3 months of observation, the changes in the structural characteristics provided clear evidence of the underlying mechanisms for their occurrence and temporal evolution.

\section{Experimental Techniques}

The subject, experimental equipment, and techniques for studying structural changes in a sample of PET film of thickness 12 microns (HOSTAPHAN ${ }^{\circledR}$ polyester films, Mitsubishi Polyester Film GmbH) irradiated with $\mathrm{Ar}^{8+}$ ions with an energy of $70 \mathrm{MeV}$ were described in [20]. In this study, we used a fluence of $2 \times 10^{12} \mathrm{ions} / \mathrm{cm}^{2}$. Irradiation was carried out in a vacuum at room temperature. The angle of ion incidence was $42^{\circ}$ with respect to the normal line to the surface. The direction of irradiation of the film coincided with the direction of motion of the film through the irradiation chamber. We used angled irradiation in order to maximize the electrical interaction of the irradiating ions along the polymer molecular chains, since it is well known that PET films and fibers have a strongly pronounced molecular texture in the direction of motion during their production [22,23].

According to our X-ray data for the pristine film [20], the ratio of the crystalline and amorphous parts of PET was 43:57, which corresponds well with the manufacturer's passport data and with the 
results of fundamental work on the study of PET structures [24]. The method for calculating this ratio via analysis of $X$-ray diffractograms was taken from [22].

X-ray images of the irradiated sample were taken immediately after irradiation. Aging of the irradiated PET film sample lasted for three months at room temperature without access to sunlight, and with periodic diffractometric observation to monitor any changes.

\section{Results and Discussion}

We previously conducted a systematic study of the effects of irradiation on the structure of PET polymer films, using swift $\mathrm{Ar}^{8+}$ ions with an energy of $70 \mathrm{MeV}$ and fluences of $2 \times 10^{10}, 5 \times 10^{11}$, and $1 \times 10^{12}$ ions $/ \mathrm{cm}^{2}$ [20]. Radiation-induced ordered regions were found in the amorphous phase of PET, using the X-ray diffraction method in the Bregg-Brentano geometry with a circular azimuthal sweep. We found that an increase in the irradiation fluence led to an increase in the areas of structural ordering, caused by the rotation of polar benzene-carboxyl subunits of repeat polymer units under the influence of the residual electric field around the ion trajectory. We found that the growth of ordering in the amorphous phase caused stresses to be transmitted along the PET molecules, deforming their crystalline regions. Thermal annealing of irradiated samples showed that the deformation of PET crystallites is elastic. We also found that irradiating samples with a fluence greater than $5 \times 10^{11}$ ions $/ \mathrm{cm}^{2}$ led to a sharp increase in the value of the atomic displacement from the equilibrium position, which we associated with the onset of the overlapping of single latent tracks.

Figure 1 shows $X$-ray diffraction patterns of the studied sample in the pristine state and after irradiation. A full description of the structural characteristics of the pristine sample was presented in [20] and can be summarized by the presence of the following characteristic areas: a halo at $2 \theta=5-15^{\circ}$ indicating the presence of an amorphous zone in the PET film under study and two isotropic azimuthal ring diffraction reflections with maxima at $2 \theta=23^{\circ}$ and $26^{\circ}$ that belong to the PET crystalline phase [25]; and a transition area in the angular range $2 \theta=15-20^{\circ}$ (see Figure 1a).
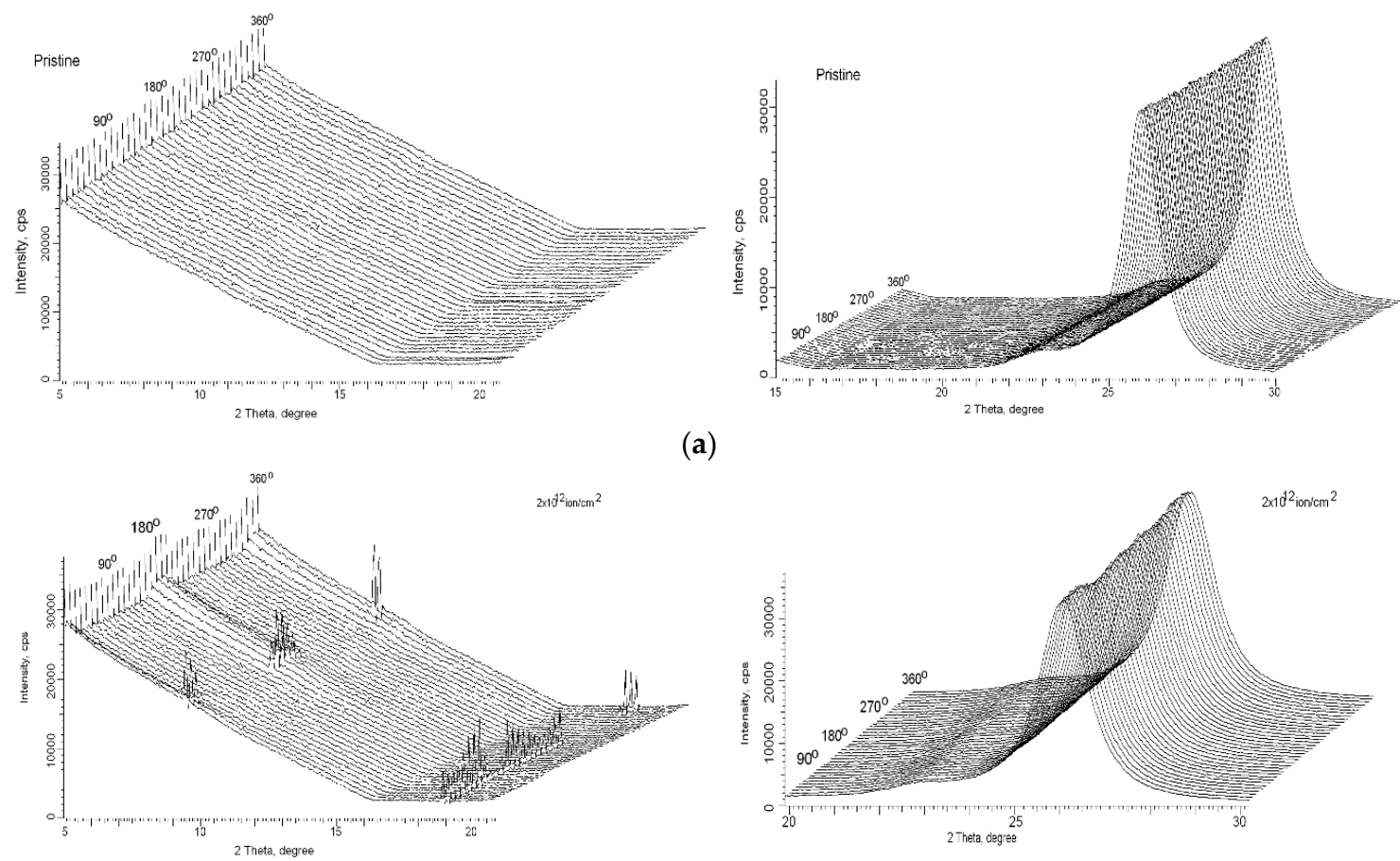

(b)

Figure 1. Cont. 

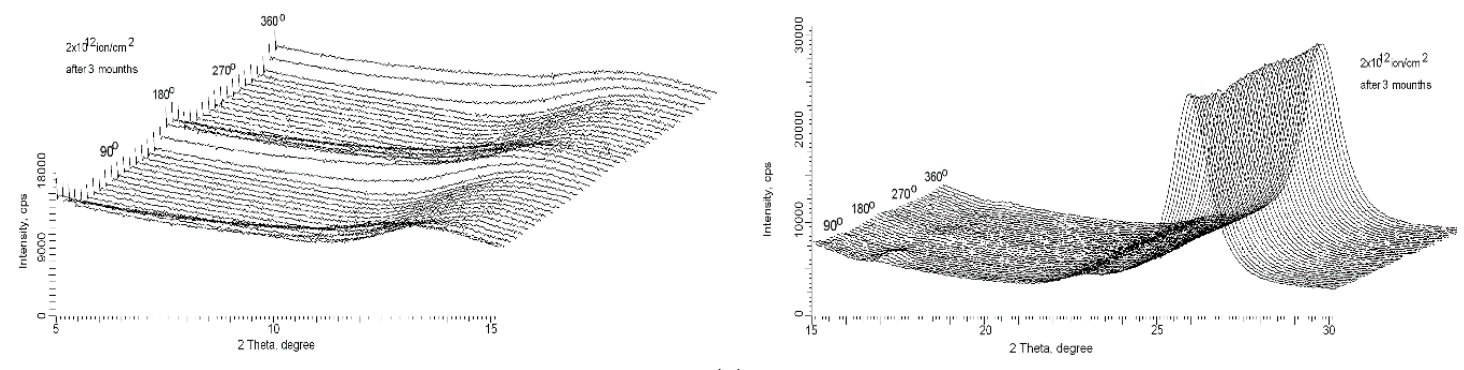

(c)

Figure 1. X-ray diffractograms of the polyethylene terephthalate (PET) sample: (a) pristine (X-ray diffractograms for the pristine sample were taken from [20] for comparison); (b) after irradiation with a dose of $2 \times 10^{12}$ ions $/ \mathrm{cm}^{2}$ in the geometry of $\varphi=0-2 \pi$; (c) temporal evolution of the diffraction spectra of the irradiated PET sample.

Figure $1 \mathrm{~b}$ shows an $\mathrm{X}$-ray reflection, typical of induced ordering, in the angular range of $2 \theta=5-12^{\circ}$ with a maximum intensity at $2 \theta=5^{\circ}$ of $24,200 \mathrm{cps}$ in the amorphous zone. This is of the same intensity and angular range as that seen for a lower irradiation fluence of $1 \times 10^{12}$ ions $/ \mathrm{cm}^{2}$ (see Figure $4 \mathrm{a}$ in [20]), showing that the growth of this induced ordering zone has stopped for both parameters. Sharp diffraction peaks have appeared at $2 \theta=9-10^{\circ}$ in the amorphous zone and $2 \theta=19^{\circ}$ in the transition zone, both with variable azimuthal intensity. The diffraction reflection rings in the crystalline phase at $2 \theta=26^{\circ}$ and $2 \theta=23^{\circ}$, which were previously azimuthally isotropic, now also show azimuthal variations in intensity, of about $10 \%$ at $2 \theta=26^{\circ}$ and rather lower at $2 \theta=23^{\circ}$.

Figure 1c shows $X$-ray diffraction patterns of the irradiated sample after 3 months of aging. The sharp diffraction peaks at $2 \theta=9-10^{\circ}$ have disappeared. Both the intensity and $2 \theta$ angular range of the induced ordering reflection in the amorphous zone have increased, but the azimuthal angular size has remained almost the same. A new broad diffraction ring has formed with a maximum about $2 \theta=13^{\circ}$ and with some azimuthal variation in intensity. The sharp diffraction peaks at $2 \theta=19^{\circ}$ were observed to move to $2 \theta=17^{\circ}$, towards the amorphous zone, diffusing and reducing in intensity but preserving the general form of their azimuthal anisotropy.

More details about azimuthal variations of all structural parts of the obtained X-ray diffraction patterns are shown in Figure 2.

The crystalline reflection rings at $2 \theta=26^{\circ}$ and $2 \theta=23^{\circ}$ have reduced in intensity with aging, showing an anti-correlation with the intensity of the induced ordering reflection in the amorphous zone, as was previously observed in [20] and attributed to the transmission of stresses along the PET chain molecules. The azimuthal variations in intensity have changed, as can be seen more clearly in Figure 2e,f below. After aging, the intensity of the diffraction ring at $2 \theta=26^{\circ}$ has a well-defined symmetry with respect to the direction $(0, \pi)$. Before aging, the intensity of the diffraction ring at $2 \theta=23^{\circ}$ was broadly isotropic except for a local maximum at $\varphi$ approximately $3 \pi / 2$. After aging the local maximum is at $\varphi$ approximately $\pi$.

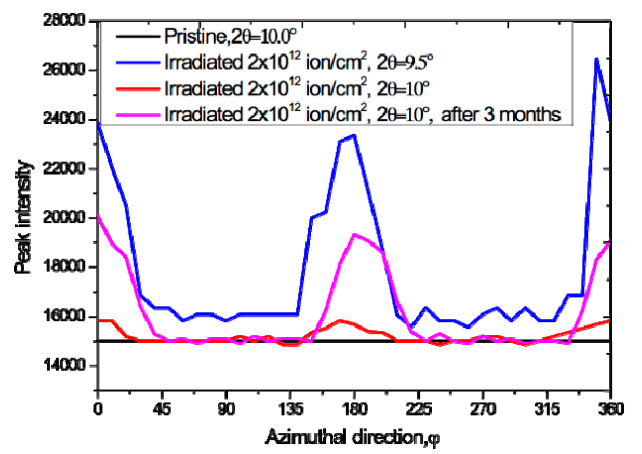

(a)

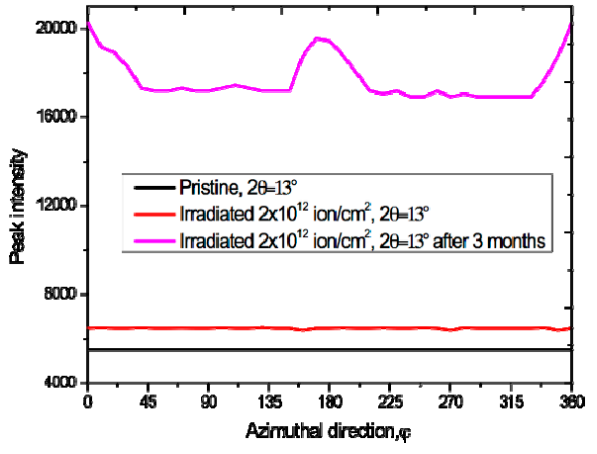

(b)

Figure 2. Cont. 


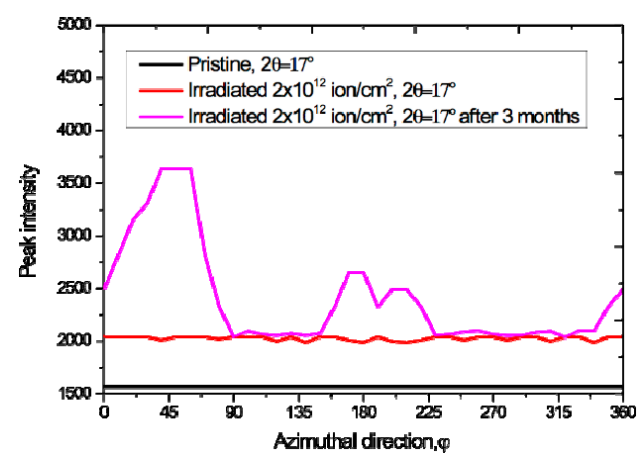

(c)

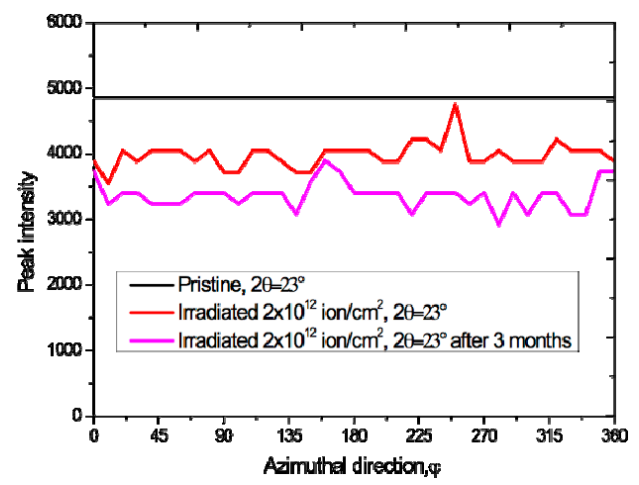

(e)

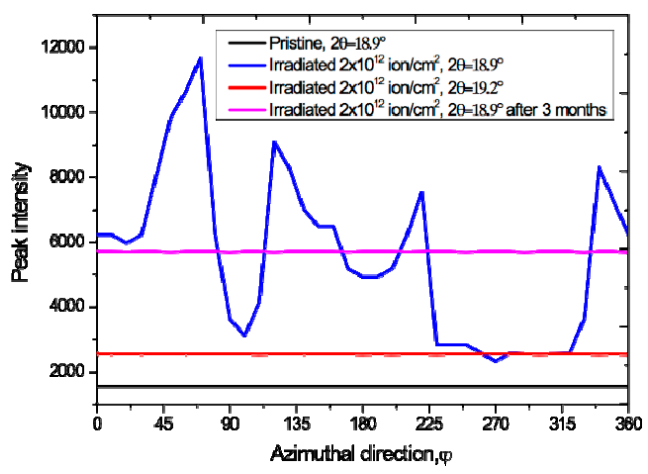

(d)

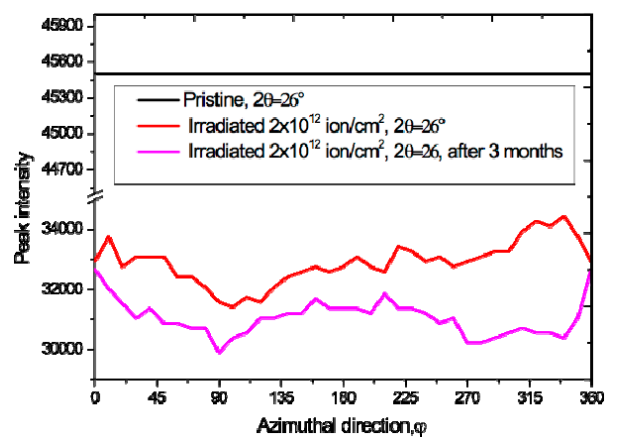

(f)

Figure 2. Azimuthal variations in the intensity of diffraction reflections after irradiation with a dose of $2 \times 10^{12}$ ions $/ \mathrm{cm}^{2}$ (red lines), and after aging for 3 months (pink lines). Pristine behavior (black lines) is included for reference: (a) $2 \theta=9-10^{\circ}$; (b) $2 \theta=13^{\circ}$; (c) $2 \theta=17^{\circ}$; (d) $2 \theta=19^{\circ}$; (e) $2 \theta=23^{\circ}$; (f) $2 \theta=26^{\circ}$.

All of the diffraction rings described above have azimuthal distributions of intensities consisting of rings on which long or short arcs with variable intensity are superimposed. The diagrams in Figure 3 show that there is some symmetry in the peak azimuthal intensities in the polar coordinate system $(2 \theta, \varphi)$. After irradiation, there is a clear oblique cross located predominantly along lines at angles of $\pi / 4$ with respect to the direction of the texture. After aging the picture is more complicated but still contains some cross-like symmetries. Such X-ray diffraction patterns are typical of solid polymer materials consisting of chain molecules coiled in spirals, in which rings due to the polycrystalline character of the material are superimposed with arcs due to the spiral configuration of the chain molecules $[22,25,26]$.

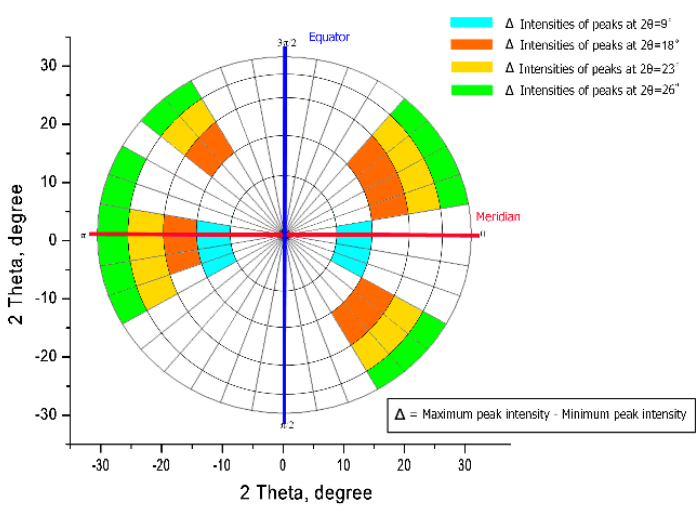

(a)

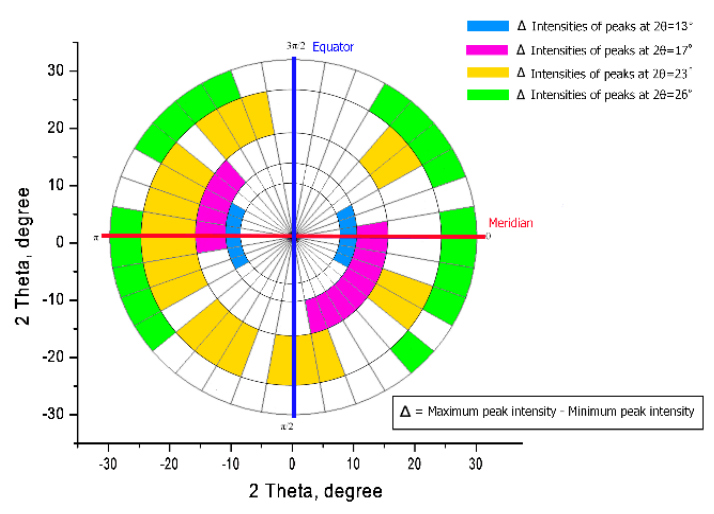

(b)

Figure 3. Diagram showing peak azimuthal intensities in the polar coordinate system $(2 \theta, \varphi)$ : (a) after irradiation and (b) after 3 months. 
X-ray diffractometry of chain molecules is a well-studied field of radiography with its own terminology and is described in a large number of major monographs (e.g., [22]). According to the terminology used in X-ray diffractometry, the term "meridian" is used in place of the term "texture direction" that we have been using to describe the direction along the axis of the chain molecule. The direction perpendicular to the meridian is referred to as the "equator". The position of arcs and spots along the meridian, equator, and/or at an oblique angle to both of them provide complete information about the parameters of the spirals formed by these chain molecules. In particular, the presence of a cross-like pattern in an X-ray diffraction pattern is a reliable confirmation of the existence of a spiral in the structure of chain molecules (e.g., see Figure 90, p. 142 in [22]). The X-ray diffraction patterns of helical polymer chain molecules are so well-understood that analysis of their details can be used to determine properties of the polymer material. For example, in [25] X-ray diffractometry of isotactic poly(methyl methacrylate) (it-PMMA), whose molecules are coiled in a double helix, was used to determine the mechanical properties of this polymer. The physics of the formation of double helical polymer chains is also well-understood as being due to dipole-dipole interactions between neighboring chain molecules together with the rotation of subunits of the repeat units of the chain molecules.

For example, in [26], the formation of a double helix in a sample of synthetic sulfonated aramid polyanion, poly-2,2' - disulphonyl-4, $4^{\prime}$ - benzidine terephthalamide (PBDT), was shown to be due to rotation of benzene rings associated with dipole units such as $-\mathrm{SO}_{3}-$ and $-\mathrm{NHCO}-$. A repeat unit of PBDT polymer molecule contains three benzene rings separated from the axis of the molecule by $\sigma$-hinges, but only two of them contain attached asymmetric dipole groups, which allows them to rotate during intermolecular interactions. In addition, the repeat PBDT unit includes a carboxyl group, which also has the ability to rotate on $\sigma$ - hinges. The authors showed the helical conformation via X-ray diffraction, NMR spectroscopy, and molecular dynamics (MD) simulations. The authors explain the emergence of a helix formation in a pre-ordered PBDT solution as due to the joint action of: intermolecular forces between functional units such as $-\mathrm{SO}_{3}{ }^{-}$and $-\mathrm{NHCO}^{-}$in neighboring chains; $\pi$-stacking; and dipole-dipole interactions, together with the rotation of each subunit within the chain PBDT molecule.

If we apply this body of knowledge to our data, we can conclude that molecular helical structures appeared in the PET film when irradiated with a fluence of $2 \times 10^{12} \mathrm{ions} / \mathrm{cm}^{2}$. These helical structures degraded with aging, in contrast to the region of induced ordering in the amorphous zone, which not only did not dissipate with aging but increased in both intensity and $2 \theta$ angular size. It is possible to understand the observed evolution of spiral structures after high irradiation fluence via the well-known fact that the overlap of the zones of influence of latent tracks increases with increasing irradiation fluence $[27,28]$. If the irradiation fluence is too low to cause overlap, the residual cylindrically symmetric electric fields inside each zone of influence act independently of each other and the dipole groups of the benzene-carboxyl subunits interact only with the residual electric field of a single track. This results in cylindrically symmetric ordering of these subunits, as shown in [20].

If the fluence is high enough to cause overlapping of latent tracks, the behavior of benzene-carboxyl subunits will be determined not only by the residual electric field of a single latent track but also by the fields of neighboring tracks. Because of the cylindrical symmetry of the latent tracks, the individual electric fields in overlapping regions act in opposition to each other to reduce the effective electric field. This, together with the fact that the benzene-carboxyl subunits are already ordered, allows dipole-dipole interactions between the subunits of neighboring chain molecules, causing correlated rotations of these subunits. The asymmetric nature of the dipoles in the subunits, together with their rotations, is sufficient in this situation for the formation of a spiral conformation of the subunits in neighboring chain molecules, as shown in [26]. As with PBDT, repeat units of PET chain molecules contain an aromatic ring with an attached dipole -CO unit. The only difference between PBDT and PET is that the repeat unit of PET contains a single benzene ring and a single type of dipole unit. We consider the explanation given by the authors of [26] to be physically well-founded and confirmed 
experimentally. In our explanation of the spiral formation effect we found, we follow the physical representations described in [26]. In our case, however, the only rotatable subunits are benzene rings, and the only cause of the interchain interaction is a dipole-dipole interaction.

It is known from the physics of the class of polymer electrets to which PET belongs that dipole-dipole interactions between neighboring chain molecules have low energy (0.02-0.9 eV) [29], which leads to low temporal stability of dipole-dipole conformational structures, as observed here.

We would like to emphasize that, as in [20], we clearly see the reversible nature of this additional ordering, in contrast to the irreversibility of the processes of radiation crosslinking caused by $\delta$-electrons and their cascades of secondary electrons, both immediately after irradiation and throughout the aging of irradiated polymer films [9,10,12].

We also note that here we are discussing an effect that appears immediately after irradiation: aging helps us understand it but it is not due to the aging itself. As we mentioned in the introduction, we are aware of no literature describing any molecular ordering occurring from the aging of polymer films irradiated by swift ions. There remains a lack of clarity in the understanding of the processes that occur in polymer films under ion irradiation. There are many different models and hypotheses describing the formation of latent tracks in solids, including polymers, such as the Coulomb explosion model, the exciton self-trapping model, the thermal soldering model, the electronic braking hypothesis of energy losses of incoming ions, etc., (see e.g., [3]). These models and hypotheses have all been formulated to explain such experimental facts in ion-irradiated polymers as polymerization, amorphization, radiolysis, change in phase composition, and chemical cross-linking. A number of models described in [3] lead to effects such as saturation of the defect concentration when latent tracks overlap. This circumstance was used in [30] to estimate the transverse dimensions of latent tracks in crystals and polymers.

In the wider context, we can make two observations about the results here and in [20]. First, reversing the arguments above, in this situation some information about the structure of the residual electric fields in the PET film is provided by the x-ray diffractograms, even though they only map positions of reflecting centers in the molecules, because these show the rotation of benzene-carboxyl units responding to a residual electric field. In other words, the benzene carboxyl subunits are acting as nanoindicators of the gradient of the electric field, but provide no information about its magnitude. This information could be provided by optical studies of irradiated films, for example, using the Franz-Keldysh effect [31], which the authors plan to do in the future. Second, our results, together with those in [26] for a very different experimental situation, suggest that preordering is a very important, and perhaps essential, prerequisite for the emergence of spiralization.

We now turn our attention to understanding the increase of the induced ordering in the amorphous zone after aging. This type of induced ordering is associated with the intrachain rotation of benzene-carboxyl subunits [20] and is as stable as the residual electric field itself. Figure 4a shows that the value of the root mean square (RMS) atomic displacement from equilibrium positions for the irradiated PET sample is almost constant over time, confirming the well-known long-term stability of latent tracks in polymers such as PET. For example, [15] shows the presence of a residual electric field in a latent track in polyamide foil after irradiation with uranium ions at a distance of up to $3 \mathrm{~nm}$ from the center of the track after 10 years of storage.

The general decrease in the intensity of the main diffraction reflection at $2 \theta=26^{\circ}$ alongside a simultaneous increase in the meridian reflection associated with the induced ordering in the amorphous zone [20] is observed over a time frame typical for creep-like effects. This leads us to suggest that not only mobile benzene-carboxyl subunits but also molecular chains themselves are involved in the post-irradiation ordering processes caused by residual electric fields. 


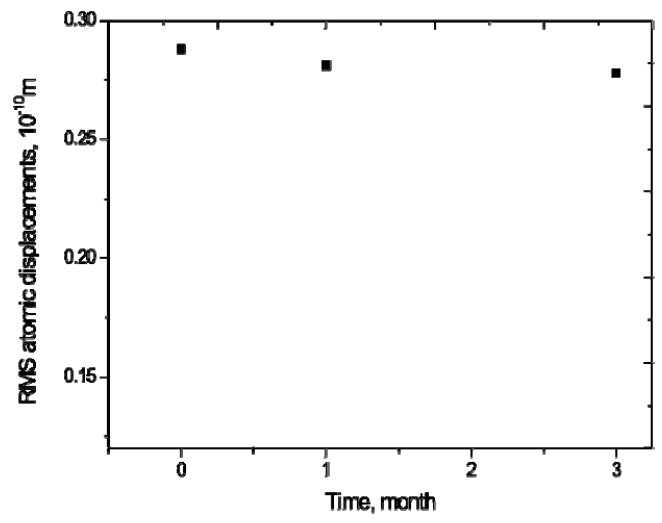

(a)

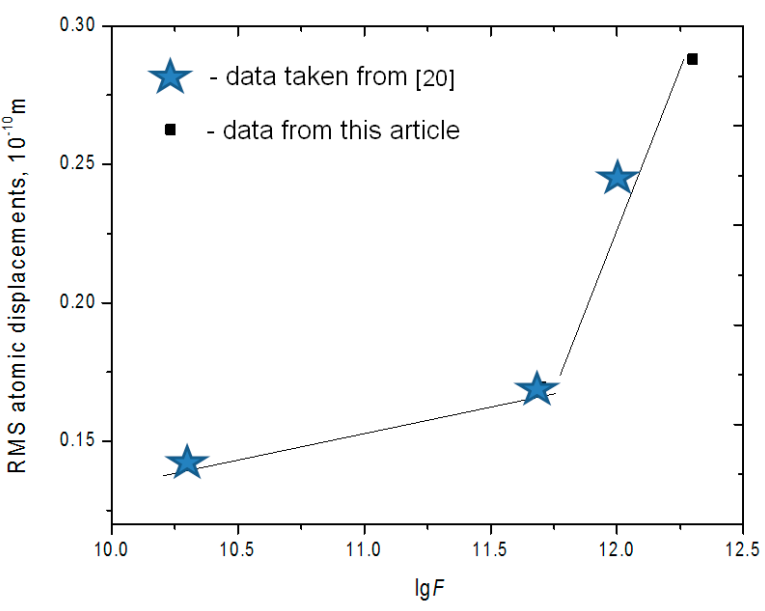

(b)

Figure 4. (a) Temporal dynamics in RMS displacement of the sample irradiated with a dose of $2 \times 10^{12}$ ions $/ \mathrm{cm}^{2}$ and (b) RMS displacement immediately after irradiation for four fluences between $5 \times 10^{10}$ and $2 \times 10^{12}$ ions $/ \mathrm{cm}^{2}$ (data for the first three taken from [20]).

Figure $4 \mathrm{~b}$ shows that the RMS atomic displacement from the equilibrium position for the highest fluence of $2 \times 10^{12}$ ions $/ \mathrm{cm}^{2}$ fits into the previously established trend of a sharp increase in RMS value for fluences above $5 \times 10^{11}$ ions $/ \mathrm{cm}^{2}$. In the case of irradiation of solid materials with a well-defined crystalline lattice, an increase in atomic displacement from the equilibrium position is usually taken as an indication of growing disorder and amorphization in the structure of the crystalline lattice. In this study, however, the conformational rotation of mobile subunits of repeat units of the chain molecules leads both to increased levels of ordering and to the large atomic displacement values.

\section{Conclusions}

The experimental results presented here and in [20] confirm the existence of induced ordering in PET caused by residual electric fields of latent tracks after irradiation with swift argon ions. Induced ordering occurs both as a result of intra-chain rotation of the mobile subunits of repeat units of the chain molecules within single latent tracks and, when the irradiating fluence is high enough to cause overlapping of latent tracks, inter-chain interaction of these mobile subunits, leading to the creation of spiral conformations of the subunits in neighboring chain molecules.

Author Contributions: Conceptualization, A.Z.T. And M.V.Z.; methodology, A.Z.T., M.V.Z., and A.L.K.; formal analysis, A.L.K. And M.V.Z.; investigation, A.Z.T., M.V.Z., and F.E.H.; resources, M.V.Z.; writing-original draft preparation, review and editing, A.Z.T., F.E.H., A.L.K., and M.V.Z.; visualization, A.Z.T. And A.L.K.; supervision, M.V.Z.; project administration, M.V.Z.; funding acquisition, M.V.Z. All authors have read and agreed to the published version of the manuscript.

Funding: This research received no external funding and Dr. F.E. Harrison took part in this research as a volunteer.

Conflicts of Interest: The authors declare no conflict of interest.

\section{References}

1. Apel, P.Y. Fabrication of functional micro-and nanoporous materials from polymers modified by swift heavy ions. Radiat. Phys. Chem. 2019, 159, 25-34.

2. Liu, F.; Wang, M.; Wang, X.; Wang, P.; Shen, W.; Ding, S.; Wang, Y. Fabrication and application of nanoporous polymer ion-track membranes. Nanotechnology 2018, 30, 052001. [CrossRef]

3. Werner, W.; Wendler, E. Ion Beam Modification of Solids; Springer Nature: Berlin/Heidelberg, Germany, 2016; Volume 61.

4. Olgun, G. Ionizing radiation: A versatile tool for nanostructuring of polymers. Pure Appl. Chem. 2016, 88, 1049-1061. 
5. Singh, L.; Samra, K.S.; Singh, R.; Solania, I.; Avasthi, D.K. Degradation of nickel (86 MeV) ion irradiated polystyrene. J. Non-Cryst. Solids 2008, 354, 41-48. [CrossRef]

6. Turos, A.; Jagielski, J.; Piątkowska, A.; Bieliński, D.; Ślusarski, L.; Madi, N.K. Ion beam modification of surface properties of polyethylene. Vacuum 2003, 70, 201-206. [CrossRef]

7. Bielinski, D.; Lipinski, P.; Slusarski, L.; Grams, J.; Paryjczak, T.; Jagielski, J.; Madi, N.K. Surface layer modification of ion bombarded HDPE. Surf. Sci. 2004, 564, 179-186. [CrossRef]

8. Abdul-Kader, A.M.; El-Gendy, Y.A.; Al-Rashdy, A.A. Improve the physical and chemical properties of biocompatible polymer material by MeV He ion beam. Radiat. Phys. Chem. 2012, 81, 798-802. [CrossRef]

9. Apel, P.; Spohr, R.; Trautmann, C.; Vutsadakis, V. Track structure in polyethylene terephthalate irradiated by heavy ions: LET dependence of track diameter. Radiat. Meas. 1999, 31, 51-56. [CrossRef]

10. Apel, P.Y.; Blonskaya, I.V.; Oganessian, V.R.; Orelovitch, O.L.; Trautmann, C. Morphology of latent and etched heavy ion tracks in radiation resistant polymers polyimide and poly (ethylene naphthalate). Nucl. Instrum. Methods Phys. Res. Sect. B 2001, 185, 216-221. [CrossRef]

11. Steckenreiter, T.; Balanzat, E.; Fuess, H.; Trautmann, C. Chemical modifications of PET induced by swift heavy ions. Nucl. Instrum. Methods Phys. Res. Sect. B 1997, 131, 159-166. [CrossRef]

12. Apel, P.Y.; Blonskaya, I.V.; Ivanov, O.M.; Kristavchuk, O.V.; Lizunov, N.E.; Nechaev, A.N.; Dmitriev, S.N. Creation of Ion-Selective Membranes from Polyethylene Terephthalate Films Irradiated with Heavy Ions: Critical Parameters of the Process. Membr. Membr. Technol. 2020, 2, 98-108. [CrossRef]

13. Tamon, K.; Barillon, R.; Yamauchi, T. Application of Radial Electron Fluence around ion tracks for the description of track response data of polyethylene terephthalate as a polymeric nuclear track detector. Nucl. Instrum. Methods Phys. Res. Sect. B 2019, 461, 260-266.

14. Maletic, S.B.; Cerovic, D.D.; Dojcilovic, J.R. A study of structural and spectral properties of ion-beam modified polyethylene terephthalate membrane. Nucl. Instrum. Methods Phys. Res. Sect. B 2019, 441, 1-7. [CrossRef]

15. Abu Saleh, S.; Eyal, Y. Porous tracks along wakes of swift uranium ions in polyimide. Appl. Phys. Lett. 2004, 85, 2529-2531. [CrossRef]

16. Wang, P.; Wang, M.; Liu, F.; Ding, S.; Wang, X.; Du, G.; Wang, Y. Ultrafast ion sieving using nanoporous polymeric membranes. Nat. Commun. 2018, 9, 1-9. [CrossRef]

17. Sessler, G.M. Physical principles of electrets. In Electrets; Springer: Berlin/Heidelberg, Germany, 1980; pp. 13-80.

18. Crawford, W.T.; Humphrey, J.S., Jr.; De Sorbo, W. Method for Making Visible Radiation Damage Tracks in Track Registration Materials. U.S. Patent No. 3,612,871, 12 October 1971.

19. Zhu, Z.; Maekawa, Y.; Liu, Q.; Yoshida, M. Influence of UV light illumination on latent track structure in PET. Nucl. Instrum. Methods Phys. Res. Sect. B 2005, 236, 61-67. [CrossRef]

20. Zdorovets, M.V.; Kozlovskiy, A.L.; Harrison, F.E.; Tuleushev, A.Z. Induced ordering in polyethylene terephthalate films irradiated with Ar ions with an energy of $70 \mathrm{MeV}$. Surf. Coat. Technol. 2020, 386, 125490. [CrossRef]

21. Eliel, E.L.; Wilen, S.H.; Doyle, M.P. Basic Organic Stereochemistry; Wiley-Interscience: New York, NY, USA, 2001.

22. Weinstein, B.K. X-ray Diffraction by Chain Molecules; Publishing House of the USSR Academy of Sciences: Moscow, Russia, 1963.

23. Misra, A.; Stein, R.S. Stress-induced Crystallization of Poly (Ethylene Terephthalate). J. Polym. Sci. Polym. Phys. Ed. 1979, 17, 235-257. [CrossRef]

24. Daubeny, R.D.P.; Bunn, C.W.; Brown, C.J. The crystal structure of polyethylene terephthalate. Proc. R. Soc. Lond. Ser. A Math. Phys. Sci. 1954, 226, 531-542.

25. Urbanek, S.; Tashiro, K.; Kitayama, T.; Hatada, K. Crystallite modulus of double-stranded helices of isotactic poly (methyl methacrylate): The X-ray measurement and the theoretical calculation. Polymer 1999, 40, 3345-3351. [CrossRef]

26. Wang, Y.; He, Y.; Yu, Z.; Gao, J.; Ten Brinck, S.; Slebodnick, C.; Ensing, B. Double helical conformation and extreme rigidity in a rodlike polyelectrolyte. Nat. Commun. 2019, 10, 1-8. [CrossRef] [PubMed]

27. García, G.; Rivera, A.; Crespillo, M.L.; Gordillo, N.; Olivares, J.; Agulló-López, F. Amorphization kinetics under swift heavy ion irradiation: A cumulative overlapping-track approach. Nucl. Instrum. Methods Phys. Res. Sect. B 2011, 269, 492-497. [CrossRef]

28. Peña-Rodríguez, O.; Manzano-Santamaría, J.; Rivera, A.; García, G.; Olivares, J.; Agulló-López, F. Kinetics of amorphization induced by swift heavy ions in $\alpha$-quartz. J. Nucl. Mater. 2012, 430, 125-131. [CrossRef] 
29. Das-Gupta, D.K. Molecular processes in polymer electrets. J. Electrost. 2001, 51, 159-166. [CrossRef]

30. Chris, T.; Schwartz, K.; Steckenreiter, T. Specificity of ion induced damage. Nucl. Instrum. Methods Phys. Res. Sect. B 1999, 156, 162-169.

31. Pankove, J.I. Optical Processes in Semiconductors; Courier Corporation: Gloucester, MA, USA, 1971.

(C) 2020 by the authors. Licensee MDPI, Basel, Switzerland. This article is an open access article distributed under the terms and conditions of the Creative Commons Attribution (CC BY) license (http://creativecommons.org/licenses/by/4.0/). 